\title{
Roles of the $\mu$-opioid receptor and its related signaling pathways in the pathogenesis of premenstrual syndrome liver-qi stagnation
}

\author{
CHUNHONG SONG and LING XUE
}

\begin{abstract}
Key Laboratory for Classical Theory of Traditional Chinese Medicine, The Education Ministry, Shandong University of Traditional Chinese Medicine, Jinan, Shandong 250355, P.R. China
\end{abstract}

Received December 28, 2015; Accepted December 23, 2016

DOI: $10.3892 /$ etm.2017.4374

\begin{abstract}
The present study aimed to investigate the roles of the $\mu$-opioid receptor (MOR) and its related signaling pathways in the pathogenesis of premenstrual syndrome (PMS) liver-qi stagnation, along with the therapeutic effects of the Shu-Yu capsule in treating the condition. A PMS liver-qi stagnation rat model was established using a chronic restraint stress method. The protein expression level of MOR within rat hippocampal tissue was detected via western blot analysis and cyclic adenosine monophosphate (cAMP) levels within the supernatant of a rat hippocampal cell culture were determined by ELISA. The western blot analysis indicated that the hippocampal expression level of MOR was significantly elevated in the PMS liver-qi stagnation model group. However, subsequent treatment with a Shu-Yu capsule was found to significantly decrease the level of MOR expression. In addition, in vitro experiments were performed, whereby primary hippocampal neurons were treated with model rat serum. It was observed that the level of MOR expression was significantly elevated, while brain-derived neurotrophic factor (BDNF) and cAMP levels in the culture supernatant were significantly decreased. These effects were reversed by treatment with serum from the Shu-Yu capsule-treated rats. Furthermore, when treated with the MOR activator DAMGO, the following were significantly decreased in the primary neurons: Phosphorylation levels of $c A M P$ response element binding protein and extracellular signal-regulated protein kinases (ERK); BDNF expression; and cAMP content in the culture supernatant. These effects were reversed in primary neurons treated with DAMGO and Shu-Yu-containing rat serum. Collectively, the data suggest that increased MOR expression and activation of the cAMP/ERK
\end{abstract}

Correspondence to: Dr Ling Xue, Key Laboratory for Classical Theory of Traditional Chinese Medicine, The Education Ministry, Shandong University of Traditional Chinese Medicine, 4655 University Road, Jinan, Shandong 250355, P.R. China

E-mail: songchunhong8069@126.com

Key words: premenstrual syndrome, liver-qi stagnation, $\mu$-opioid receptor, Shu-Yu capsule, cyclic adenosine monophosphate/extracellular signal-regulated kinases signaling pathway signaling pathway in the hippocampus may be involved in the pathogenesis of PMS liver-qi stagnation. Furthermore, the efficacy of the Shu-Yu capsule in treating the condition may be via its regulation of MOR receptor signaling.

\section{Introduction}

Premenstrual syndrome (PMS) is a prevalent condition that occurs in women of a reproductive age, and is experienced in $>75 \%$ of females aged 15-49 years old (1). In addition to the physical symptoms, including headaches, edema, lumbago, breast pain, and fatigue, cases of severe PMS cases are also associated with substantial mood changes, including anxiety, depression, suicidal ideation and aggressive behavior (2). However, the pathophysiological causes of PMS remain unclear. In traditional Chinese medicine, liver-qi stagnation is recognized as a major secondary condition of PMS, for which treatment with the Shu-Yu capsule is considered to be beneficial (3). PMS liver-qi stagnation is a syndrome induced by abnormal flow of liver qi, which is characterized by premenstrual depression, chest tightness and sighing. A previous study concerning 526 PMS females from the Shandong province, China, has indicated a liver-qi stagnation incidence of $27.5 \%$ (4). In traditional Chinese medicine, PMS liver-qi stagnation is mainly treated through relieving the depressed liver qi, and nourishing pi and dredging qi.

Increasing evidence indicates that the $\mu$-opioid receptor (MOR) serves a key role in the regulation of mood and emotional disorders (5-7). In particular, it has been observed that withdrawal of endogenous opioid peptides is associated with the development of PMS, and decreased levels of opioid peptides are considered as a marker of the condition (8-13). Furthermore, the effects of Vitex agnus-castus methanol extracts in the treatment of PMS seem to depend on the activation of MOR (14). Collectively, these findings suggest that MOR and its ligands may be associated with the pathophysiology of PMS.

Previous studies by our group have found that the level of serum endomorphin (EM), which is a highly agonistic and selective endogenous agonist for MOR, are significantly elevated in PMS rat models, indicating the involvement of MOR in PMS development (15-17). However, the role of MOR in PMS pathophysiology, along with the corresponding molecular mechanisms, it not well understood. In the present 
study, a PMS liver-qi stagnation rat model was established by a chronic restraint stress method. This rat model, along with a primary hippocampal neuron culture, were used to evaluate the pathophysiology of PMS and the potential involvement of MOR, whereby the expression levels of MOR and its related proteins were detected via ELISA and western blot analysis. Furthermore, the effects of the Shu-Yu capsule on PMS liver-qi stagnation and MOR signaling were investigated.

\section{Materials and methods}

Ethics statement. All animal experiments were conducted according to the ethical guidelines of the Ethics Committee of Shandong University of Traditional Chinese Medicine (Jinan, China).

Animal model establishment and grouping. A total of 50 non-pregnant, specific pathogen-free female Wistar rats, weighing 150-180 g and aged 5-6 weeks, were purchased from the Vital River Laboratory Animal Technology Co., Ltd. (Beijing, China; lot no. 2012052307409). These animals were maintained in cages, with a reversed 12-h light/dark cycle (lights on at 9:00 p.m.; lights off at 9:00 a.m.), with free access to water prior to the experiments. The rats were maintained at a temperature of $22-26^{\circ} \mathrm{C}$ and at a relative humidity of $50-70 \%$.

The rats were randomly divided into control, PMS liver-qi stagnation model, and Shu-Yu treatment groups $(n=10$ per group). Shu-Yu capsules, which mainly consisted of white peony root, bupleurum, cyperus tuber and licorice root, were purchased from Yangguang Haichuan Pharmaceutical Science and Technology Development Co., Ltd. (investigational new drug approval no. 2008L11169; Qingdao, China). An in vivo model of PMS liver-qi stagnation was established in the PMS liver qi stagnation method rats and the Shu-Yu treatment group using the chronic restraint stress method, as previously described (18). In the treatment group, model rats were subjected to daily administration (9:00 a.m.), of a Shu-Yu capsule via gavage, at a dose of $0.41 \mathrm{~g} / \mathrm{kg}$ body weight ( $\sim 8$ times the clinical dosage), over the modeling period. Rats in the control and model groups received sterile water at a dose of $10 \mathrm{ml} / \mathrm{kg}$ body weight over the same period. In order to assess behavioral alterations, rats were stimulated by glass rod pricking and behavior was judged subjectively.

Primary hippocampal neuron culture. Primary hippocampal neurons for culture were obtained from $~ 100$ neonatal Wistar rats (Experimental Animal Center, Shandong University of Traditional Chinese Medicine; male:female, 1:1; weight, 5-7 g) within $24 \mathrm{~h}$ of birth. The rats were maintained in the conditions described above until they were sacrificed using decollation and their brains were removed. Briefly, the hippocampi were separated under sterile conditions and digested with $0.25 \%$ trypsin at $37^{\circ} \mathrm{C}$ for $20 \mathrm{~min}$. Following filtering with a 200-mesh filter, the cells were seeded into poly-lysine-coated 6 -well plates at a density of $4 \times 10^{5} / \mathrm{ml}$, then cultured in a $37^{\circ} \mathrm{C}$, $5 \% \mathrm{CO}_{2}$ incubator. After $24 \mathrm{~h}$, the cells were incubated at $37^{\circ} \mathrm{C}$ with serum-free Neurobasal-A medium (10888-022; Gibco; Thermo Fisher Scientific, Inc., Waltham, MA, USA), which was replenished every 3 days. After 7 days, the cells were cultured with Neurobasal-A medium (10888-022; Gibco;
Thermo Fisher Scientific, Inc.) containing 10\% fetal bovine serum (FBS; 10099-133; Gibco; Thermo Fisher Scientific, Inc.) and B-27 supplement (17504-044; Gibco; Thermo Fisher Scientific, Inc.) for $24 \mathrm{~h}$ at $37^{\circ} \mathrm{C}$.

Serum preparation and cell culture treatment. To prepare serum from the model rats, the rats were sacrificed via decapitation following modeling and blood serum samples were collected. The serum was inactivated in a $56^{\circ} \mathrm{C}$ water bath for $30 \mathrm{~min}$ and sterilized with a $0.45-\mathrm{mm}$ membrane filter. Then, $200 \mu \mathrm{l}$ doses of serum (10\% V/V) from the control, model and treatment groups were added to each well to incubate the cultured primary hippocampal neurons for $24 \mathrm{~h}$ at $37^{\circ} \mathrm{C}$. An additional blank group of cells received no treatment.

For the preparation of drug-containing serum, 30 normal male Wistar rats (Experimental Animal Center, Shandong University of Traditional Chinese Medicine), weighing 140-160 g, were administered with sterile water $(10 \mathrm{ml} / \mathrm{kg}$ body weight/day), a Shu-Yu capsule ( $0.41 \mathrm{~g} / \mathrm{kg}$ body weight/day) and naloxone (10 g/kg body weight/day; lot no. H20064789; Beijing Kawin Biotech, Beijing, China) for 5 days. The rats were maintained in the same conditions described above. Following the last drug administration (50 min), a 6-ml blood sample was collected via the inferior vena cava following an intraperitoneal injection of $1 \%$ sodium pentobarbital and the rats were then sacrificed by decollation. Serum was obtained by centrifugation at $1,680 \times \mathrm{g}\left(4^{\circ} \mathrm{C}\right)$ for $15 \mathrm{~min}$. Then, $200 \mu \mathrm{l}$ serum $(10 \% \mathrm{~V} / \mathrm{V})$ was added to each well for incubation of the primary neurons at $37^{\circ} \mathrm{C}$ for $24 \mathrm{~h}$.

In addition, for the treatment of the MOR activator, DAMGO (E7384; Sigma-Aldrich; Merck Millipore, Darmstadt, Germany), primary neurons were incubated, in triplicates, with the above-mentioned drug-containing serum at $37^{\circ} \mathrm{C}$ for $24 \mathrm{~h}$, then treated with $1 \mu \mathrm{mol} / 1 \mathrm{DAMGO}$ per well for $0,5,15,30,45$ and $60 \mathrm{~min}$.

Western blot analysis. The expression levels of MOR and brain-derived neurotrophic factor (BDNF) and the phosphorylation levels of extracellular signal-regulated kinases (ERK) and cyclic adenosine monophosphate response element-binding protein (CREB) were detected via western blot analysis. Total protein was obtained from the hippocampi of the neonatal rats following sacrifice and the primary neuron culture. Protein concentration was determined using an enhanced BCA protein concentration assessment kit (P0010; Beyotime Institute of Biotechnology, Haimen, China), according to the manufacturer's instructions. A total of $150 \mu \mathrm{l}$ pre-iced RIPA lysis buffer (P0013B) containing 1\% PMSF (ST506; both Beyotime Institute of Biotechnology) was added into each well to lyse the cells on ice for $30 \mathrm{~min}$. Then, the solution was transferred into a centrifuge tube, which was subjected to the centrifugation at $10,800 \times \mathrm{g}\left(4^{\circ} \mathrm{C}\right)$ for $5 \mathrm{~min}$. The supernatant was kept and stored until further use. A total of $50 \mu \mathrm{g}$ protein was loaded per lane and separated with 10\% SDS-PAGE and then transferred onto a nitrocellulose membrane. The membrane was blocked with Tris-buffered saline with Tween (TBST) containing 5\% fat-free milk at room temperature for $1 \mathrm{~h}$, then incubated with rabbit anti-rat anti-MOR polyclonal antibody (1:200 dilution; ab10275; Abcam, Cambridge, MA, USA), anti-BDNF polyclonal antibody (1:1,000 dilution; ab46176; 
Abcam), anti-CREB monoclonal antibody (1:800 dilution; 9197; Cell Signaling Technology, Inc., Danvers, MA, USA), anti-phospho-CREB monoclonal antibody (1:800 dilution; 9198; Cell Signaling Technology, Inc.), anti-ERK polyclonal antibody (1:900 dilution; 9102s; Cell Signaling Technology, Inc.), anti-phospho-ERK polyclonal antibody (1:800 dilution; 9101s; Cell Signaling Technology, Inc.) and anti- $\beta$-actin monoclonal antibody, as an internal control (1:3,000 dilution; A1978; Sigma-Aldrich; Merck Millipore), at room temperature for $2 \mathrm{~h}$. The membrane was then incubated with goat anti-rabbit secondary antibody (1:1,800 dilution; SB-200; Sungene Biotech, Tianjin, China) at room temperature for $1 \mathrm{~h}$ and washed with TBST. The membrane was developed using electrochemiluminescence (ECL kit; P90720; Merck Millipore), according to the manufacturer's instructions, and the bands were analyzed with ImageJ software v.1.48u (National Institutes of Health, Bethesda, MA, USA).

Enzyme-linked immunosorbent assay (ELISA). The cyclic adenosine monophosphate (cAMP) level in the culture supernatant was detected with an ELISA kit (F-15181; Xitang Biotechnology Co., Ltd., Shanghai, China), according to the manufacturer's instructions.

Statistical analysis. Data are presented as the mean \pm standard deviation. Statistical analysis was performed with GraphPad Prism 5.0 software (GraphPad Software, Inc., La Jolla, CA USA) and the one-way analysis of variance test was used for comparison of the means. $\mathrm{P}<0.05$ was considered to indicate a statistically significant difference.

\section{Results}

Behavior alterations in PMS liver-qi stagnation rats were reversed by Shu-Yu capsule treatment. A PMS liver-qi stagnation rat model was established using a chronic restraint stress method. The behavior observations indicated that, compared with control rats, PMS liver-qi stagnation rats were lethargic, and had coarse hair and dull eyes. According to subjective assessment, when stimulated with glass rod pricking electric current, the rats in the control group reacted swiftly, while the model rats' response was slower. From the open-field test, it was found that the scores of rats in the model group were significantly decreased relative to the control group $(\mathrm{P}<0.01)$. Following treatment with the Shu-Yu capsule, the treatment group scores were significantly increased relative to the untreated model group $(\mathrm{P}<0.05$; Fig. 1$)$.

Increased hippocampal MOR expression in PMS liver-qi stagnation rats was reversed by Shu-Yu capsule treatment. To investigate the expression levels of MOR in the hippocampus of PMS liver-qi stagnation rats, a western blot analysis was performed. The results indicated that, relative to the control group, the expression of MOR in the hippocampus was significantly increased in the PMS liver-qi stagnation model group $(\mathrm{P}<0.01)$. In addition, hippocampal MOR expression was significantly decreased in model rats following treatment with the Shu-Yu capsule $(\mathrm{P}<0.01 ;$ Fig. 2). These results indicate that Shu-Yu capsule treatment significantly decreased the expression of MOR in the hippocampus of PMS liver-qi stagnation model rats.

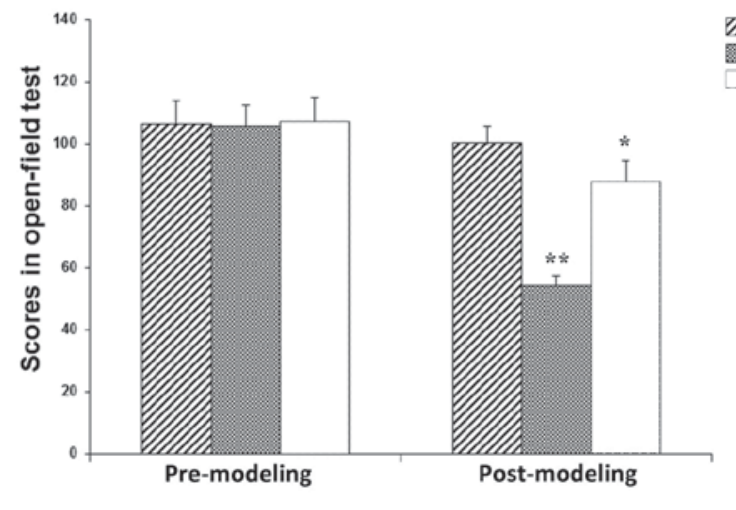

Figure 1. Open-field test of PMS liver-qi stagnation model rats. Pre- and post-modeling open-field test scores of rats from the control, Model and Treatment groups. ${ }^{*} \mathrm{P}<0.05$ vs. the Model group, ${ }^{* *} \mathrm{P}<0.01$ vs. the control group. PMS, premenstrual syndrome; Model, PMS liver-qi stagnation group; Treatment, Shu Yu capsule treatment group.

Shu-Yu capsule treatment reverses the altered hippocampal levels of MOR, BDNF and cAMP induced by model rat serum. The effects of model rat serum on the expression of MOR and BDNF in the primary hippocampal neurons and on cAMP levels in the culture supernatant were evaluated by western blot analysis (Fig. 3). Primary hippocampal neurons were treated with rat serum from the control, model and treatment groups, while cells in the blank group received no treatment. Results from western blotting indicated that there was no significant difference in MOR and BDNF expression between the blank cells and primary neurons treated with normal rat serum $(\mathrm{P}>0.05)$. For the primary neurons treated with model rat serum, levels of MOR were significantly elevated $(\mathrm{P}<0.05)$, while levels of BDNF were significantly lower $(\mathrm{P}<0.05)$ than in primary neurons treated with normal rat serum. These effects appeared to be reversed by treatment with the Shu-Yu capsule treatment group serum $(\mathrm{P}<0.05$; Fig. $3 \mathrm{~A}$ and $\mathrm{B})$. Furthermore, results of the ELISA indicated that there was no significant difference in the cAMP level of culture supernatant between the blank cells and the cells treated with normal rat serum $(\mathrm{P}>0.05)$. Meanwhile, the level of cAMP in the culture supernatant from cells treated with model rat serum was significantly lower than that in cells treated with normal rat serum $(\mathrm{P}<0.05)$, with this level significantly elevated for cells treated with the Shu-Yu capsule treatment group serum relative to the model rat serum-treated rats $(\mathrm{P}<0.05$; Fig. $3 \mathrm{C})$. Collectively, these results suggest that, when cultured with the serum from model rats, MOR expression is increased and BDNF expression decreased in primary hippocampal neurons, while the cAMP level in the culture supernatant is decreased. However, these effects were reversed by incubation with the serum from the Shu-Yu capsule treatment group.

Effects of MOR activation on CREB and ERK phosphorylation, BDNF expression, and cAMP level are reversed by Shu-Yu treatment. To evaluate the role of MOR in PMS liver-qi stagnation pathophysiology, primary neurons were treated with the MOR activator, DAMGO. A western blot analysis was then performed to detect the phosphorylation of CREB and ERK, the expression of BDNF in the cells and the cAMP level in the culture supernatant. In order to determine the appropriate 
A

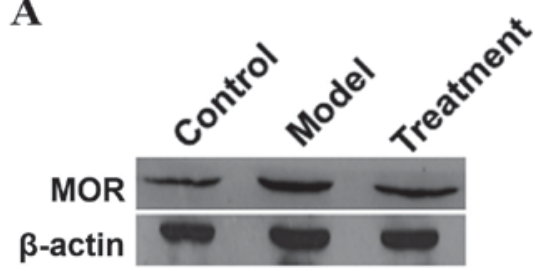

B

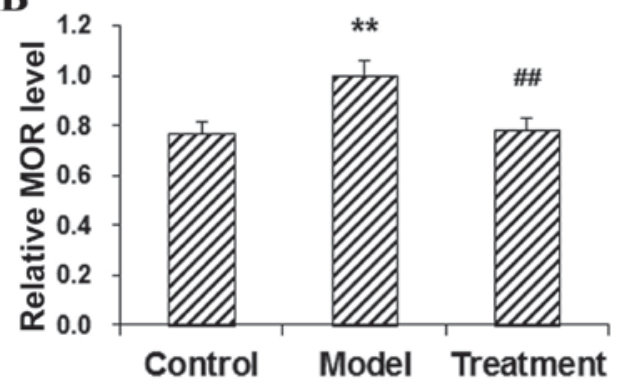

Figure 2. Expression of MOR in the hippocampi of PMS liver-qi stagnation model rats. (A) Levels of MOR protein in the hippocampi of rats from the control, PMS liver-qi stagnation model and Shu-Yu capsule treatment groups were detected by western blot analysis. (B) Protein expression levels of MOR, as detected by western blotting, were quantified by ImageJ analysis. ${ }^{* *} \mathrm{P}<0.01$ vs. the control group; ${ }^{\# \#} \mathrm{P}<0.01$ vs. the model group. MOR, $\mu$-opioid receptor; PMS, premenstrual syndrome; Model, PMS liver-qi stagnation group; Treatment, Shu Yu capsule treatment group.

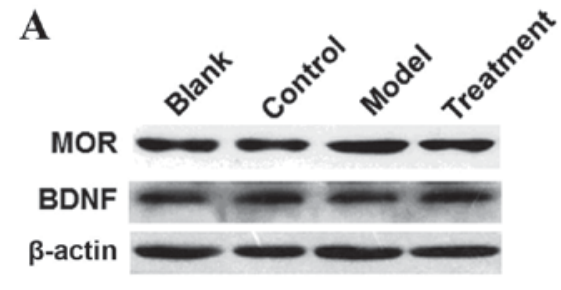

C

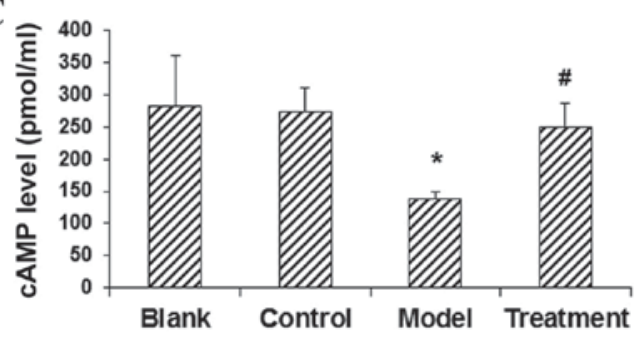

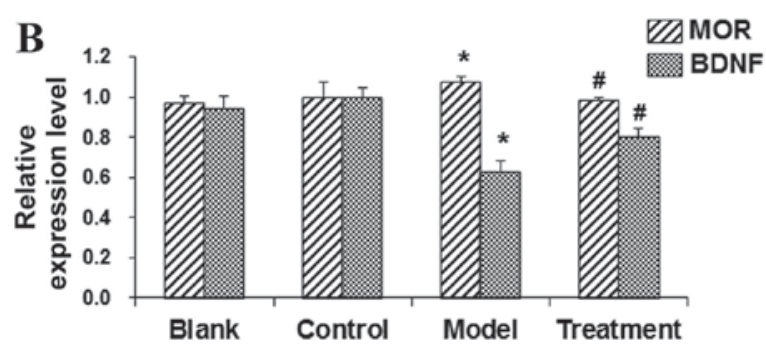

Figure 3. Effects of model rat serum on the levels of MOR, BDNF, and cAMP in primary hippocampal neurons. Primary hippocampal neurons were treated with rat serum from the control, Model and Treatment groups, while cells in the blank group received no treatment. (A) Protein expression levels of MOR and BDNF in the primary hippocampal neurons were detected by western blot analysis. (B) The expression levels of MOR and BDNF, as detected by western blotting, were quantified by ImageJ analysis. (C) cAMP levels in the hippocampal culture supernatant were detected by ELISA. * $<0.05$ vs. the control group, ${ }^{\#} \mathrm{P}<0.05$ vs. the model group. MOR, $\mu$-opioid receptor; BDNF, brain-derived neurotrophic factor; cAMP, cyclic adenosine monophosphate; Model, PMS liver-qi stagnation group; Treatment, Shu Yu capsule treatment group.

treatment period, primary neurons were treated with $1 \mu \mathrm{mol} / 1$ DAMGO for $0,5,15,30,45$ and $60 \mathrm{~min}$, and the phosphorylation level of ERK was detected. The results indicated that 15-30 min was the most effective treatment duration for DAMGO (Fig. 4); therefore, a 20 min treatment duration was used in the following experiments. It was observed via western blotting that cells treated with the activator alone and the cells treated with activator plus normal rat serum had significantly decreased phosphorylation levels of CREB and ERK, and decreased levels of BDNF expression, relative to blank control cells $(\mathrm{P}<0.05)$. By contrast, in cells treated with the activator and Shu-Yu-containing serum, the phosphorylation levels of CREB and ERK, and BDNF expression, were significantly increased relative to cells treated with the activator plus normal rat serum $(\mathrm{P}<0.05)$. Similar effects were observed in cells treated with the activator and naloxone-containing serum $(\mathrm{P}<0.05$; Fig. 5A-C).

In the evaluation of cAMP level, it was observed that cAMP levels in the culture supernatant from cells treated with
DAMGO or DAMGO plus normal rat serum was significantly decreased relative to the control cells $(\mathrm{P}<0.01)$. In turn, cAMP was significantly increased in cells treated with DAMGO plus Shu-Yu- or naloxone-containing serum $(\mathrm{P}<0.01$ and $\mathrm{P}<0.05$, respectively; Fig. 5D). Collectively, these results suggest that MOR activation may decrease hippocampal CREB and ERK phosphorylation, hippocampal BDNF expression and cAMP level in hippocampal culture supernatant, and that these effects were reversed by the administration of serum from Shu-Yu capsule-treated rats.

\section{Discussion}

In the present study, it was observed that MOR expression was significantly elevated within the hippocampal region of PMS liver-qi stagnation model rats, an effect that could be significantly inhibited by treatment with the Shu-Yu capsule. A previous study by the current authors in PMS liver-qi invasion rats has indicated that the level of MOR expression is decreased 


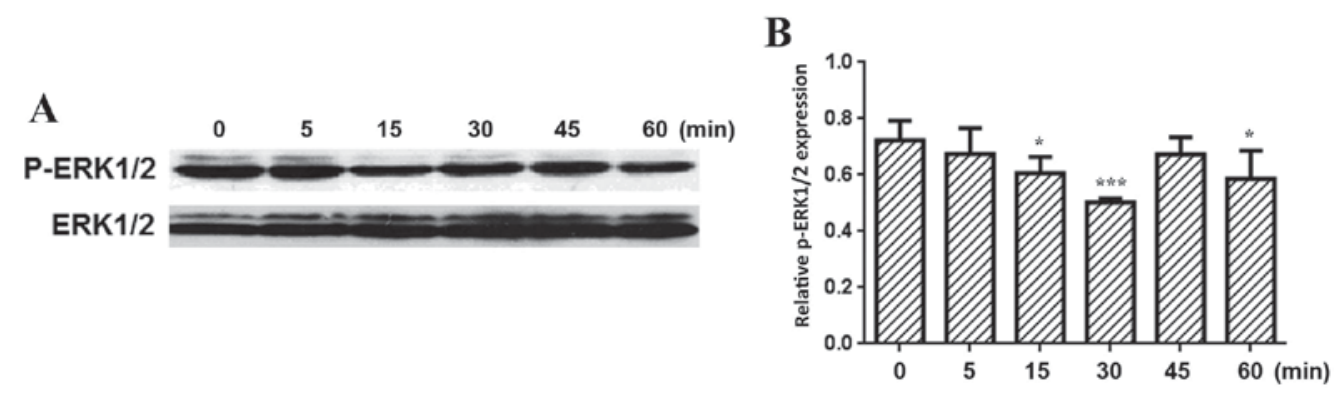

Figure 4. Effects of DAMGO on ERK phosphorylation in primary hippocampal neurons. Primary neurons were treated with $1 \mu \mathrm{mol} / 1 \mathrm{DAMGO}$ for $0,5,15,30$, 45 and $60 \mathrm{~min}$ (A) Levels of p-ERK1/2 and total ERK1/2 were detected by western blot analysis. (B) Relative phosphorylation levels of ERK1/2 in the primary neurons. ${ }^{*} \mathrm{P}<0.05$ and ${ }^{* * *} \mathrm{P}<0.001$ vs. 0 min. DAMGO, activator of $\mu$-opioid receptor; p-ERK1/2, phosphorylated ERK1/2.
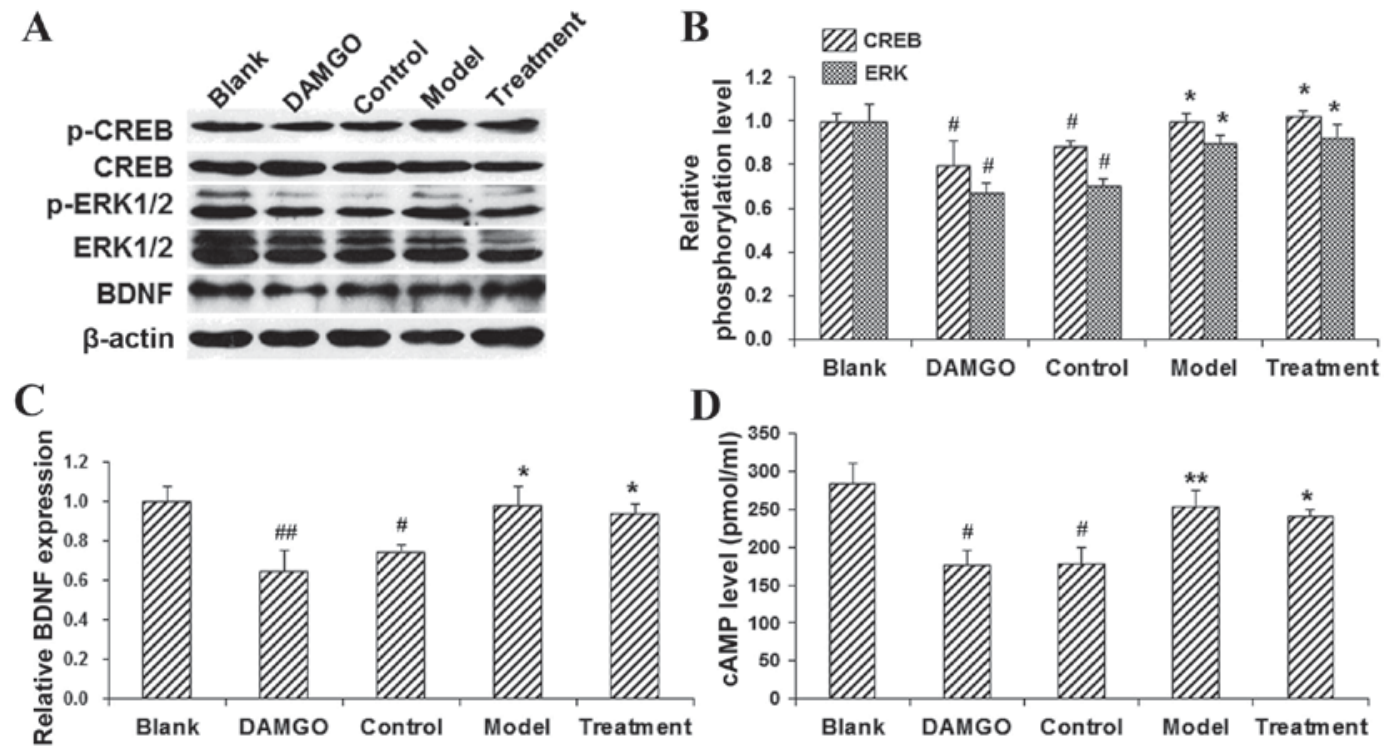

\section{D}

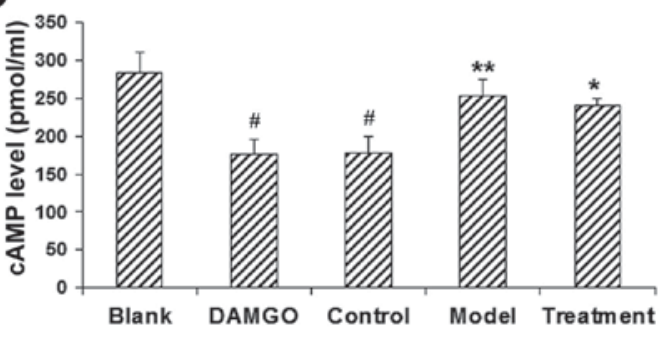

Figure 5. Effects of DAMGO on CREB and ERK phosphorylation, BDNF expression and cAMP levels in serum-treated primary hippocampal neurons. Primary neurons were first incubated with serum from the control, model and treatment groups for $24 \mathrm{~h}$, then treated with $1 \mu \mathrm{mol} / 1 \mathrm{DAMGO}$ for $20 \mathrm{~min}$. The blank group received no treatment and cells in the DAMGO group were treated with DAMGO alone. (A) CREB and ERK phosphorylation and BDNF expression in the primary hippocampal neurons were detected by western blot analysis. (B) Levels of CREB and ERK phosphorylation, as detected by western blotting, were quantified by ImageJ analysis (C) The expression of BDNF, as detected by western blotting, was quantified by ImageJ analysis (D) cAMP levels in the hippocampal culture supernatant were detected by ELISA. ${ }^{*} \mathrm{P}<0.05$ and ${ }^{\# *} \mathrm{P}<0.01$ vs. the blank group, ${ }^{*} \mathrm{P}<0.05$ and ${ }^{* *} \mathrm{P}<0.01$ vs. the control group. DAMGO, activator of $\mu$-opioid receptor; cAMP, cyclic adenosine monophosphate; CREB, cAMP response element-binding protein; ERK, extracellular signal-regulated kinases; BDNF, brain-derived neurotrophic factor; p-, phosphorylated; Model, PMS liver-qi stagnation group; Treatment, Shu-Yu capsule treatment group.

in the parietal and frontal cortex, while the receptor expression is increased in the hypothalamus and hippocampus (19). Collectively, these observations suggest that increased MOR expression within the hippocampus may be associated with the pathogenesis of PMS liver-qi stagnation.

The neurobiological factors involved in the development of PMS are considered to be similar to those involved in the development of severe depression $(20,21)$. In rats, it has been observed that a non-selective opioid receptor antagonist was able to alter the time in suspension during forced swimming (22), which improved depressive-like behavior $(17,23)$. In the present study, MOR and its related signaling pathways were investigated in rat primary hippocampal neurons. This was achieved via the treatment of cultured cells with DAMGO, followed by treatment with serum from Shu-Yu-treated and -untreated model rats. The results indicated that relative to the control group, MOR expression was increased, while
BDNF expression was decreased, in the model serum treatments; an effect which could be reversed by administration of serum from the Shu-Yu treatment group. Furthermore, DAMGO was able to decrease hippocampal CREB and ERK phosphorylation, hippocampal BDNF expression and levels of cAMP in the hippocampal culture supernatant. These effects were reversed by administration of serum from the Shu-Yu treatment group.

As a potential mechanism, MOR may bind to and inactivate adenylate cyclase, by binding to the Gi/o and Gz subunits of the G-protein coupled receptor, thus inhibiting the production of cAMP and decreasing the expression of downstream proteins $(24,25)$. Protein kinase A may be activated by cAMP, with active PKA then phosphorylating and activating CREB, leading to transcriptional regulation of the CREB target genes, including BDNF. This would result in increased BDNF binding to its receptor, tropomyosin receptor kinase $\mathrm{B}$, and 
initiation of the phospholipase $\mathrm{Cy}$, Ras-mitogen-activated protein kinase and phosphoinositide 3-kinase pathways (26). In addition, BDNF potentially exerts antidepressant effects through the Ras-Raf- mitogen-activated protein/extracellular signal-regulated kinase kinase (MEK)-ERK pathway (27). Under physiological conditions, ERK is the only downstream substrate of MEK to be identified, with activation of ERK potentially inducing the phosphorylation of various transcription factors and regulating the transcriptional expression of immediate early genes, including c-Fos, c-Myc, c-Jun and Egrl (28).

The downstream effects of CREB also have implications for PMS. Phosphorylation and activation of CREB regulates the transcription of various cytokines, thereby modulating cell regeneration and survival (29). In addition, CREB serves an important role in the regulation of synaptic growth (30) and in the maintenance and modulation of learning, memory function and emotions $(31,32)$. In the neurons of patients with depression, disruptions in the cAMP signaling pathway and decreased mRNA expression levels of CREB have been observed in the frontal lobe. This was alleviated by increasing the intracellular concentration of CREB with antidepressant drugs (33).

The present study found that BDNF expression was decreased in the PMS rat model. In previous studies, decreased BDNF levels have been found in the brains of depressed patients, with an increase in BNDF potentially exerting antidepressant effects $(34,35)$. Furthermore, Liu et al (36) have observed that in rats with depression-like behavior, the level of BDNF mRNA was significantly decreased in the hippocampus, particularly in the CA1 and CA3 regions, indicating that BDNF may be a potential target for antidepressant treatment. It has been indicated that current antidepressants and mood stabilizers may act on the ERK signaling pathway, which regulates synaptic plasticity in the central nervous system (37). In addition, the ERK signaling pathway is considered to be involved in the modulation of depression. Feng et al (38) found that in a mouse model of depression, levels of phospho-ERK1/2 and ERK2 were significantly reduced in the frontal cortex and hippocampus, indicating that the absence of the ERK signaling pathway may be associated with depression-like behavior in mice. These findings indicate that ERK signaling may be involved in the effects of Shu-Yu capsule treatment on PMS liver-qi stagnation in vivo and in vitro. However, further studies are necessary in order to determine which of the Shu-Yu capsule components have the observed therapeutic effects in PMS liver-qi stagnation.

The present study did have some limitations. In order to investigate the modulating mechanism of the Shu-Yu capsule on PMS liver-qi stagnation, the Shu-Yu capsule-containing serum was used to treat the cells. However, the individual effects of the active ingredient(s) in the capsule remain unclear. In future research, experimental groups should be treated with the active ingredient(s) of the Shu-Yu capsule in order to investigate the therapeutic effects of the active ingredient(s) on PMS liver-qi stagnation.

In conclusion, the present results indicated that $\mathrm{Shu}-\mathrm{Yu}$ capsule treatment may decrease MOR expression within the hippocampi of PMS liver-qi stagnation model rats. Treatment with model rat serum was found to increase MOR and decrease BDNF expression in cultured primary hippocampal neurons, while also decreasing cAMP levels in the culture supernatant. Furthermore, the activation of MOR appeared to decrease hippocampal CREB and ERK phosphorylation, BDNF expression and culture supernatant cAMP levels. Such effects were reversed by administration of serum from Shu-Yu capsule-treated model rats. Collectively, these observations suggest that increased hippocampal MOR expression and cAMP/ERK signaling are involved in the pathogenesis of PMS liver-qi stagnation, with the Shu-Yu capsule exerting its therapeutic effects via the regulation of MOR signaling. These findings may aid in understanding the mechanisms behind PMS liver-qi stagnation therapeutics.

\section{Acknowledgements}

The present study was supported by the National Natural Science Fund Project, China (grant no. 81173162).

\section{References}

1. Halbreich U, Borenstein J, Pearlstein T and Kahn LS: The prevalence, impairment, impact, and burden of premenstrual dysphoric disorder (PMS/PMDD). Psychoneuroendocrinology 28 (Suppl 3): S1-S23, 2003.

2. Kelderhouse K and Taylor JS: A review of treatment and management modalities for premenstrual dysphoric disorder. Nurs Womens Health 17: 294-305, 2013.

3. Yu J, Qiao MQ and Zhang HY: Treatment of Premenstrual Syndrome. Zhong Guo Mei Tan Gong Ye Yi Xue Za Zhi 5: 433-435, 2005 (In Chinese).

4. Qiao M, Zhang Z, Xu X, et al: Epidemiologic study on the distribution of premenstrual syndromes. Zhong Guo Zhong Yi Ji Chu Za Zhi 3: 31-33, 1997 (In Chinese).

5. Morales-Mulia M, Estrada-Camarena E, Amaya MI, Mejía-Mauríes S, Sollozo-Dupont I, Mengod G and de Gortari P: Anxiolytic effects of ethanol are partially related to a reduced expression of adenylyl cyclase 5 but not to $\mu$-opioid receptor activation in rat nucleus accumbens. Behav Brain Res 235: 189-194, 2012.

6. Komatsu H, Ohara A, Sasaki K, Abe H, Hattori H, Hall FS, Uhl GR and Sora I: Decreased response to social defeat stress in mu-opioid-receptor knockout mice. Pharmacol Biochem Behav 99: 676-682, 2011.

7. Johnston CE, Herschel DJ, Lasek AW, Hammer RP Jr and Nikulina EM: Knockdown of ventral tegmental area mu-opioid receptors in rats prevents effects of social defeat stress: Implications for amphetamine cross-sensitization, social avoidance, weight regulation and expression of brain-derived neurotrophic factor. Neuropharmacology 89: 325-334, 2015.

8. Halbreich U and Endicott J: Possible involvement of endorphin withdrawal or imbalance in specific premenstrual syndromes and postpartum depression. Med Hypotheses 7: 1045-1058, 1981.

9. Tulenheimo A, Laatikainen $\mathrm{T}$ and Salminen K: Plasma beta-endorphin immunoreactivity in premenstrual tension. Br J Obstet Gynaecol 94: 26-29, 1987.

10. Giannini AJ, Martin DM and Turner CE: Beta-endorphin decline in late luteal phase dysphoric disorder. Int J Psychiatry Med 20: 279-284, 1990.

11. Giannini AJ, Melemis SM, Martin DM and Folts DJ: Symptoms of premenstrual syndrome as a function of beta-endorphin: Two subtypes. Prog Neuropsychopharmacol Biol Psychiatry 18: 321-327, 1994.

12. Chuong CJ, Coulam CB, Kao PC, Bergstralh EJ and Go VL: Neuropeptide levels in premenstrual syndrome. Fertil Steril 44: 760-765, 1985.

13. Chuong CJ and Hsi BP: Effect of naloxone on luteinizing hormone secretion in premenstrual syndrome. Fertil Steril 61: 1039-1044, 1994.

14. Webster D, Lu J, Chen SN, Farnsworth N and Wang ZJ: Activation of the mu-opiate receptor by Vitex agnus-castus methanol extracts: Implication for its use in PMS. J Ethnopharmacol 106: 216-221, 2006.

15. Ma J and Zhang HY: Study on the content of endomorphin in the hypothalamus and serum of premenstrual syndrome model rats. Pharmaceutical and Clinical Research 16: 343-346, 2008. 
16. Wang $\mathrm{F}$, Xue L and Zhang $\mathrm{H}$ : Influence of jingqianping granule on expression of $\mu$ opium receptor in the hypothalamus and hippocampus of premenstrual syndrome rats with liver Qi reversion. J Trad Chin Med 15: 22, 2011.

17. Wang FR, Qiao MQ, Xue L and Wei S: Possible Involvement of $\mu$ opioid receptor in the antidepressant-like effect of shuyu formula in restraint stress-induced depression-like rats. Evid Based Complement Alternat Med 2015: 452412, 2015.

18. Sheng W, Haiping W and Mingqi Q: Behavioral observation and analysis of PMS Liver-qi depression rat model established by chronic restraint stress and resident-intruder paradigm. World Sci Technol 14: 1848-1852, 2012.

19. Wang FR, Xue L and Zhang HY: Effects of jingqianping granule on mRNA and protein expression of mu opioid receptor in premenstrual syndrome gan-qi invasion rats. Zhongguo Zhong Xi Yi Jie He Za Zhi 32: 1275-1279, 2012 (In Chinese).

20. Gingnell M, Comasco E, Oreland L, Fredrikson M and Sundstrom-Poromaa I: Neuroticism-related personality traits are related to symptom severity in patients with premenstrual dysphoric disorder and to the serotonin transporter gene-linked polymorphism 5-HTTPLPR. Arch Womens Ment Health 13 417-423, 2010.

21. Cunningham J, Yonkers KA, O'Brien S and Eriksson E: Update on research and treatment of premenstrual dysphoric disorder. Harv Rev Psychiatry 17: 120-137, 2009.

22. Haj-Mirzaian A, Ostadhadi S, Kordjazy N, Dehpour AR and Ejtemaei Mehr S: Opioid/NMDA receptors blockade reverses the depressant-like behavior of foot shock stress in the mouse forced swimming test. Eur J Pharmacol 735: 26-31, 2014.

23. Lutz PE, Ayranci G, Chu-Sin-Chung P, Matifas A, Koebel P, Filliol D, Befort K, Ouagazzal AM and Kieffer BL: Distinct mu, delta and kappa opioid receptor mechanisms underlie low sociability and depressive-like behaviors during heroin abstinence. Neuropsychopharmacology 39: 2694-2705, 2014.

24. Belcheva MM, Tan Y, Heaton VM, Clark AL and Coscia CJ: $\mathrm{Mu}$ opioid transactivation and down-regulation of the epidermal growth factor receptor in astrocytes: Implications for mitogen-activated protein kinase signaling. Mol Pharmaco 64 1391-1401, 2003

25. Liu Q, Ye CY and Zhang DC: Effects of crosstalk between opioid receptors and MAP kinases on opioid tolerance and dependence. Zhong Guo Yao Wu Yi Lai Za Zhi 3: 165-168, 2003 (In Chinese).

26. Yamashita M, Shinnakasu R, Asou H, Kimura M, Hasegawa A Hashimoto K, Hatano N, Ogata M and Nakayama T: Ras-ERK MAPK cascade regulates GATA3 stability and Th2 differentiation through ubiquitin-proteasome pathway. J Biol Chem 280 29409-29419, 2005.

27. Yamashita M, Shinnakasu R, Asou H, Kimura M, Hasegawa A, Hashimoto K, Hatano N, Ogata M and Nakayama T: Ras-ERK MAPK cascade regulates GATA3 stability and Th2 differentiation through ubiquitin-proteasome pathway. J Biol Chem 280 29409-29419, 2005
28. Chambard JC, Lefloch R, Pouyssegur J and Lenormand P: ERK implication in cell cycle regulation. Biochim Biophys Acta 1773 1299-1310, 2007.

29. Tabuchi A, Sakaya H, Kisukeda T, Fushiki H and Tsuda M: Involvement of an upstream stimulatory factor as well as cAMP-responsive element-binding protein in the activation of brain-derived neurotrophic factor gene promoter I. J Biol Chem 277: 35920-35931, 2002.

30. Dworkin S, Heath JK, deJong-Curtain TA, Hogan BM, Lieschke GJ, Malaterre J, Ramsay RG and Mantamadiotis T: CREB activity modulates neural cell proliferation, midbrain-hindbrain organization and patterning in zebrafish. Dev Biol 307: 127-141, 2007.

31. Mao F and Yao M: Relationship between CREB and learning. Journal of Medical Forum 29: 126-127, 2008.

32. Gao D, Han M, Jiang X and Sun X: The effects of repeatedly forced swim stress on hippocampal neurogenesis and p-CREB expression in rats. Nervous Diseases and Mental Health 10: 329-331, 2010.

33. Ligeza A, Wawrzczak-Bargiela A, Kaminska D, Korostynski M and Przewlocki R: Regulation of ERK1/2 phosphorylation by acute and chronic morphine-implications for the role of cAMP-responsive element binding factor (CREB)-dependent and Ets-like protein-1 (Elk-1)-dependent transcription; small interfering RNA-based strategy. FEBS J 275: 3836-3849, 2008.

34. Tian J: Therapeutic effects of the brain-derived neurotrophic factor on central nervous system diseases. Shandong Medical Journal 50: 112-113, 2010.

35. Hong W, Fang Y and Wang Z: Brain-derived neurotrophic factor and depression. Chinese Journal of Nervous and Mental Diseases 33: 702-703, 2007.

36. Liu S, Ren Y, Yang H, Zhao X, Liu W, Qi Z, Yang A and Zhang K: Association analysis between BDNF gene polymorphism and the anxiety symptoms of depressive disorder in Chinese Han population. Chinese Journal of Behavioral Medical and Brain Science 18: 772-774, 2009.

37. Wang L, Peng D, Xie B, Jiang K and Fang Y: The extracellular signal-regulated kinase pathway may play an important role in mediating antidepressant-stimulated hippocampus neurogenesis in depression. Med Hypotheses 79: 87-91, 2012.

38. Feng P, Guan Z, Yang X and Fang J: Impairments of ERK signal transduction in the brain in a rat model of depression induced by neonatal exposure of clomipramine. Brain Res 991: 195-205, 2003. 